\title{
The weight of half-integral weight modular forms with few non-vanishing coefficients $\bmod l$
}

by

Dohoon Choi (Seoul) and Timothy Kilbourn (Urbana, IL)

1. Introduction and statement of results. Much is known about the divisibility of the Fourier coefficients of modular forms of integral weight. When the weight is half-integral, the situation is not as clear. The distribution of the coefficients of half-integral weight modular forms in congruence classes has been studied by, among others, Balog, Darmon, and Ono [4], Ono and Skinner [11], [12], Bruinier [5], Bruinier and Ono [6], and Ahlgren and Boylan [1], [2].

Let $S_{k / 2}\left(\Gamma_{0}(N), \chi\right)$ be the space of cusp forms of weight $k / 2$ on the congruence subgroup $\Gamma_{0}(N)$ with Dirichlet character $\chi$. (See Chapter 3 in [10] for the basic definitions of a cusp form.) Recently, Ahlgren and Boylan proved the following theorem:

Theorem 1 ([2, Theorem 2]). Suppose that we have the following hypotheses:

(i) $\lambda \geq 2$ is an integer, $N$ is a positive integer with $4 \mid N$, and $\chi$ is a real Dirichlet character modulo $N$.

(ii) $l \geq 5$ is a prime such that $l \nmid N$.

(iii) $F(z)$ is a half-integral weight cusp form such that

$$
F(z)=\sum_{n=1}^{\infty} a(n) q^{n} \in S_{\lambda+1 / 2}\left(\Gamma_{0}(N), \chi\right) \cap \mathbb{Z}[[q]],
$$

where $q:=e^{2 \pi i z}$.

(iv) $F(z) \not \equiv 0(\bmod l)$, and there are finitely many square-free integers $n_{1}, \ldots, n_{t}$ such that

$$
F(z) \equiv \sum_{i=1}^{t} \sum_{m=1}^{\infty} a\left(n_{i} m^{2}\right) q^{n_{i} m^{2}}(\bmod l) .
$$

2000 Mathematics Subject Classification: Primary 11F33; Secondary 11P83. 
Write $\lambda=\bar{\lambda}+i_{\lambda}(l-1)$ with $0 \leq \bar{\lambda} \leq l-2$. Then the following are true:

(1) If $l \nmid n_{i}$ for some $i$, then

$$
\bar{\lambda} \leq 2 i_{\lambda}+1
$$

(2) If $l \mid n_{i}$ for all $i$ and $\bar{\lambda} \leq(l-3) / 2$, then

$$
\bar{\lambda} \leq 2 i_{\lambda}-\frac{l-1}{2} .
$$

(3) If $l \mid n_{i}$ for all $i$ and $\bar{\lambda} \geq(l-1) / 2$, then

$$
\bar{\lambda} \leq 2 i_{\lambda}+\frac{l+3}{2} .
$$

They used these bounds to prove Newman's conjecture [9] for the partition function for prime-power moduli. This has been extended to powers of the generating function for the partition function by the second author [8]. Here we obtain an improvement on these bounds when $l \mid n_{i}$ for all $i$.

THEOREM 2. Suppose we have the same notation and hypotheses as in Theorem 1 , and that $l \mid n_{i}$ for all $i$. Then:

(1) If $\bar{\lambda} \leq(l-3) / 2$, then

$$
\bar{\lambda} \leq i_{\lambda}-\frac{l+1}{2}
$$

(2) If $\bar{\lambda} \geq(l-1) / 2$, then

$$
\bar{\lambda} \leq i_{\lambda}+\frac{l-1}{2} .
$$

These inequalities are sharp. For example, define the quadratic character $\chi_{D}:=\left(\frac{D}{\cdot}\right)$. Let

$$
\begin{aligned}
& f(z):=\eta^{l}(24 z)=q^{l} \prod_{n=1}^{\infty}\left(1-q^{24 n}\right)^{l} \equiv \sum_{n=1}^{\infty} \chi_{12}(n) q^{l n^{2}}(\bmod l) \\
& g(z):=\eta^{l^{2}}(24 z)=q^{l^{2}} \prod_{n=1}^{\infty}\left(1-q^{24 n}\right)^{l^{2}} \equiv \sum_{n=1}^{\infty} \chi_{12}(n) q^{l^{2} n^{2}}(\bmod l) .
\end{aligned}
$$

Using the fact that $\eta(24 z) \in S_{1 / 2}\left(\Gamma_{0}(576), \chi_{12}\right)$, we deduce that $f(z) \in$ $S_{(l-1) / 2+1 / 2}\left(\Gamma_{0}(576), \chi_{12}\right)$ and $g(z) \in S_{\left(l^{2}-1\right) / 2+1 / 2}\left(\Gamma_{0}(576), \chi_{12}\right)$. For $f(z)$, we have $\bar{\lambda}=(l-1) / 2$ and $i_{\lambda}=0$, and the inequality of Theorem 2 reads $(l-1) / 2 \leq 0+(l-1) / 2$. Likewise, for $g(z)$, we have $\bar{\lambda}=0$ and $i_{\lambda}=$ $(l+1) / 2$, and the inequality is $0 \leq 0$.

2. Modular forms mod $l$. Throughout this section, we fix an integer $N \geq 1$ and a prime $l \nmid N$. The theory of modular forms modulo $l$ was developed by Serre [13] and Swinnerton-Dyer [14] for forms of level 1. We will 
recall the definitions and results that will be needed for the next section. For proofs when $N \geq 4$ and $l \nmid N$, see Gross [7].

Denote by $M_{k}$ the space of modular forms of weight $k$ on $\Gamma_{1}(N)$ with integer coefficients. If $f \in M_{k}$, let $\tilde{f} \in(\mathbb{Z} / l \mathbb{Z})[[q]]$ be the (coefficient-wise) reduction of $f \bmod l$. We write $\widetilde{M}_{k}:=\left\{\widetilde{f} \mid f \in M_{k}\right\}$ for the space of weight $k$ modular forms modulo $l$ with level $N$.

The filtration $\omega(\widetilde{f})$ of a modular form $\widetilde{f} \in \widetilde{M}_{k}$ is defined to be

$$
\omega(\widetilde{f}):=\inf \left\{k^{\prime} \mid \widetilde{f} \in \widetilde{M}_{k^{\prime}}\right\} .
$$

If $f \in M_{k}$, we sometimes write $\omega(f)$ instead of $\omega(\widetilde{f})$ if no confusion can arise. Note that $\omega(\widetilde{f})=-\infty$ if and only if $f \equiv 0(\bmod l)$. If $f \in M_{k}, g \in M_{k^{\prime}}$, and $f \equiv g \not \equiv 0(\bmod l)$, then we must have $k \equiv k^{\prime}(\bmod l-1)$. From this it follows that $\omega(\widetilde{f}) \equiv k(\bmod l-1)$.

The operator $U_{l}$ acts on $q$-expansions by

$$
\sum_{n} a(n) q^{n} \mid U_{l}=\sum_{n} a(\ln ) q^{n}
$$

Note that if $T_{l}$ denotes the $l$ th Hecke operator on $M_{k}$, then $f\left|T_{l} \equiv f\right| U_{l}$ $(\bmod l)$, so $U_{l}$ acts on the spaces $\widetilde{M}_{k}$.

We record some basic properties of the filtration of a modular form modulo $l$.

LeMma 1 ([13], [14], [7]). Let $f \in \widetilde{M}_{k}$ be such that $f \not \equiv 0(\bmod l)$. Write $k=\bar{k}+i_{k}(l-1)$ with $0 \leq \bar{k} \leq l-2$. Then:

(1) $\omega(f) \geq 0$.

(2) If $f$ is not a constant modulo $l$, then $\omega(f)>0$.

(3) $\omega(f) \equiv \omega\left(f \mid U_{l}\right)(\bmod l-1)$.

(4) $\omega\left(f^{l}\right)=l \cdot \omega(f)$.

(5) $\bar{k} \leq \omega(f) \leq k$.

3. Proof of Theorem 2. Let $F$ be as in the hypotheses. Recall the definition of the usual theta function

$$
\theta(z):=1+2 \sum_{n=1}^{\infty} q^{n^{2}} \in M_{1 / 2}\left(\Gamma_{0}(4), \chi_{-4}\right) .
$$

We define an integral weight modular form $G(z)$ by

$$
G(z):=F(z) \theta^{l}(z) \in S_{\lambda+(l+1) / 2}\left(\Gamma_{0}(N), \chi \chi-4\right) .
$$

By combining hypothesis (iv) with the fact that $l \mid n_{i}$ for all $i$, we see that

$$
F(z) \equiv \sum_{i=1}^{t} \sum_{m=1}^{\infty} a\left(l n_{i}^{\prime} m^{2}\right) q^{l n_{i}^{\prime} m^{2}}(\bmod l)
$$


and hence

$$
\left(G(z) \mid U_{l}\right)^{l} \equiv G(z)(\bmod l) .
$$

This implies that the two forms have the same filtration, and by part (4) of Lemma 1,

$$
\omega(G)=l \cdot \omega\left(G \mid U_{l}\right)
$$

First suppose $\bar{\lambda} \leq(l-3) / 2$. By hypothesis (iv) and the fact that $G(z)$ is a cusp form, we have $\omega(G)>0$ by Lemma 1(1),(2). Combining assertions (3) and (5) of Lemma 1, we get the following inequality:

$$
l \cdot\left(\bar{\lambda}+\frac{l+1}{2}\right) \leq l \cdot \omega\left(G \mid U_{l}\right)=\omega(G) \leq i_{\lambda}(l-1)+\bar{\lambda}+\frac{l+1}{2} .
$$

Solving for $\bar{\lambda}$ yields

$$
\bar{\lambda} \leq i_{\lambda}-\frac{l+1}{2}
$$

proving (1).

Next suppose $\bar{\lambda} \geq(l-1) / 2$. By the same arguments we find the inequality

$$
l \cdot\left(\bar{\lambda}+\frac{l+1}{2}-(l-1)\right) \leq l \cdot \omega\left(G \mid U_{l}\right)=\omega(G) \leq i_{\lambda}(l-1)+\bar{\lambda}+\frac{l+1}{2},
$$

and hence

$$
\bar{\lambda} \leq i_{\lambda}+\frac{l-1}{2} .
$$

This proves (2), and hence Theorem 2 .

We can also improve Corollaries 3 and 4 in [2].

Corollary. If $F(z)$ has the form

$$
F(z) \equiv \sum_{i=1}^{t} \sum_{m=1}^{\infty} a\left(n_{i} m^{2}\right) q^{n_{i} m^{2}}(\bmod l),
$$

then we must have $\bar{\lambda} \leq 2 i_{\lambda}+1$. In particular, if $\lambda \leq l-2$, then $\lambda \in\{0,1\}$.

Note that under the additional restriction $\lambda \leq l-2$, this Corollary demonstrates that Conjecture B in [2] is true. Ahlgren and Boylan have shown [3] that this Conjecture is false in general.

\section{References}

[1] S. Ahlgren and M. Boylan, Arithmetic properties of the partition function, Invent. Math. 153 (2003), 487-502.

[2] - - - Coefficients of half-integral weight modular forms modulo $l^{j}$, Math. Ann. 331 (2005), 219-239.

[3] -, 一, Addendum: "Coefficients of half-integral weight modular forms modulo $l^{j}$ " [Math. Ann. 331 (2005), 219-239], ibid., 241-242. 
[4] A. Balog, H. Darmon and K. Ono, Congruence for Fourier coefficients of halfintegral weight modular forms and special values of L-functions, in: Analytic Number Theory, Vol. 1 (Allerton Park, IL, 1995), Progr. Math. 138, Birkhäuser Boston, Boston, MA, 1996, 105-128.

[5] J. H. Bruinier, Nonvanishing modulo l of Fourier coefficients of half-integral weight modular forms, Duke Math. J. 98 (1999), 595-611.

[6] J. H. Bruinier and K. Ono, Coefficients of half-integral weight modular forms, J. Number Theory 99 (2003), 164-179.

[7] B. H. Gross, A tameness criterion for Galois representations attached to modular forms $(\bmod p)$, Duke Math. J. 61 (1990), 445-517.

[8] T. Kilbourn, The distribution of powers of the partition function modulo $l^{j}$, preprint, 2006.

[9] M. Newman, Periodicity modulo $m$ and divisibility properties of the partition function, Trans. Amer. Math. Soc. 97 (1960), 225-236.

[10] K. Ono, The web of modularity: arithmetic of the coefficients of modular forms and q-series, CBMS Reg. Conf. Ser. in Math. 102, Amer. Math. Soc., Providence, RI, 2004.

[11] K. Ono and Ch. Skinner, Fourier coefficients of half-integral weight modular forms modulo l, Ann. of Math. (2) 147 (1998), 453-470.

[12] - - - Corrigendum: "Fourier coefficients of half-integral weight modular forms modulo l", ibid. (2) 148 (1998), 361.

[13] J.-P. Serre, Formes modulaires et fonctions zêta p-adiques, in: Modular Functions of One Variable, III (Proc. Internat. Summer School, Univ. Antwerp, 1972), Lecture Notes in Math. 350, Springer, Berlin, 1973, 191-268.

[14] H. P. F. Swinnerton-Dyer, On l-adic representations and congruences for coefficients of modular forms, in: Modular Functions of One Variable, III (Proc. Internat. Summer School, Univ. Antwerp, 1972), Lecture Notes in Math. 350, Springer, Berlin, 1973, 1-55.

School of Mathematics, KIAS

207-43 Cheongnyangni 2-dong

Seoul 130-722, Korea

E-mail: choija@postech.ac.kr
Department of Mathematics University of Illinois Urbana, IL 61801, U.S.A. E-mail: tkilbour@math.uiuc.edu

Received on 25.9.2006

and in revised form on 27.11.2006 